\title{
Retrospective analysis of incidental non-trauma associated findings in severely injured patients identified by whole-body spiral CT scans
}

\author{
Johannes KM Fakler ${ }^{*}$, Orkun Özkurtul and Christoph Josten
}

\begin{abstract}
Background: Whole-body Computed Tomography (CT) scan today is considered a crucial imaging technique in the diagnostic work-up of polytrauma patients implicating a potential survival benefit. Apart from prompt identification of life threatening injuries this imaging technique provides an additional benefit by diagnosing incidental non-trauma associated medical diseases. These incidental findings might be also life threatening and warrant urgent therapy. The downside of whole-body $C T$ is a relatively high radiation exposure that might result in an increased life time cancer risk. The aim of this study was to investigate the frequency and type of non trauma associated incidental medical findings in relation to patient age and potential clinical relevance.

Methods: Between January $1^{\text {st }} 2011$ and December 15th 2012, a total of 704 trauma patients were referred to our hospital's emergency room that triggered trauma room alarm according to our trauma mechanism criteria. Of these 534 (75.8\%) received a whole-body CT according to our dedicated multiple trauma protocol. Incidental Findings (IF) were assigned in three groups according to their clinical relevance. Category 1: IF with high medical relevance (urgent life threatening conditions, unless treated) needing early investigations and intervention prior to or shortly after hospital discharge. Category 2: IF with intermediate or low medical relevance, warranting further investigations. Category 3: IF without clinical relevance.
\end{abstract}

Results: Overall 231 IFs (43.3\%) were identified, 36 (6.7\%) patients had IFs with a high clinical relevance, 48 (9.0\%) with a moderate or minor clinical relevance and 147 (27.5\%) with no clinical relevance. The distribution of incidental findings with high or moderate relevance according to age showed an incidence of $2.6 \%, 6.6 \%$ and $8.8 \%$ for patients younger than 40 years, 40 to 60 years and older than 60 years, respectively.

Conclusion: Whole-body CT scans of trauma patients demonstrate a high rate of incidental findings. Potentially life-threatening, medical findings were found in approximately every 15th patient, predominantly aged over 40 years and presenting with minor to moderate injuries and an Injury Severity Score (ISS) of 10 or less.

Keywords: Whole-body CT, Multiple injured, Polytrauma, Incidental findings

\section{Background}

Whole-body Computed Tomography (CT) scan today is considered a crucial imaging technique in the diagnostic work-up of polytrauma patients [1-3]. The multidetector approach in particular provides appropriate diagnostic algorithms for detecting relevant trauma findings with a high grade of sensitivity and specificity. This can be

\footnotetext{
*Correspondence: johannes.fakler@medizin.uni-leipzig.de

Department of Orthopaedic and Orthopaedic Trauma Surgery,

Reconstructive Surgery, University Hospital of Leipzig AöR, Liebigstr. 20,

Leipzig 04105, Germany
}

explained by recent technical improvements in CT scanner hard- and software delivering high-quality images of various body parts within a short time [4-6]. While several studies so far have demonstrated the technical feasibility of whole-body CT introducing various protocols and contrast media application techniques $[7,8]$, the exact role and clinical value of a whole-body CT in terms of nontrauma-associated additional findings, as compared to potential estimated radiation risks, is still a matter of debate. A recent multi-center trial suggested that the integration of whole-body CT into early trauma care significantly 
- frontal collision with intrusion by more than $50-75 \mathrm{~cm}$ when compared to those undergoing non-whole-body CT [1]. The downside of whole-body CT is the relatively high radiation exposure, which has led to further debates about the value of this approach especially in younger patients or elderly patients with minor injuries [9]. Although the clinical significance of incidental findings on whole-bodyCT has been reported, the success of trauma physicians in managing these findings, especially under consideration of mild trauma in elderly patients, has not been studied yet $[10,11]$. The radiation issue is pertinent because CT examinations result in much higher organ doses than those with conventional single-film $\mathrm{x}$-rays [12]. The radiation dose from whole-body CT is $10-20 \mathrm{mSv}$, which results in an estimated lifetime cancer mortality for 45-year-old people of about one in 1250 or $0.08 \%$ [13]. There is also concern that CT is replacing physical examination, and a previous study [14] with even more liberal criteria than in today's report was heavily criticised for unnecessary radiation exposure [15]. The indiscriminate use of CT for patients with minor injuries is not justified. The cohort reported by Huber-Wagner did not seem to be minimally injured. The aim of our study was to investigate the usefulness of whole-body CT for trauma assessment under consideration of non-trauma-associated age related medically relevant incidental findings and its clinical consequences.

\section{Methods}

All patients included in this study provided written informed consent for participation. The Ethical Review Board of the University of Leipzig does not require a formal IRB review for retrospective studies, and the IRB approval was therefore waived for this study. Between January $1^{\text {st }} 2011$ and December 15th 2012, a total of 704 trauma patients were referred to our hospital's emergency room that triggered trauma room alarm according to our list of trauma room activation criteria:

- $\quad$ systolic blood pressure below $90 \mathrm{mmHg}$ after trauma

- penetrating injuries to the neck and torso regions

- gunshot wounds to the neck and torso regions

- GCS below 9 after trauma

- respiratory impairment/requirement for intubation after trauma

- Fracture of more than 2 proximal bones

- Unstable chest

- Pelvic fractures

- Amputation injury proximal to hands/feet

- Spinal cord injury

- Open head wounds

- Burns $>20 \%$ and degree $\geq 2 b$

- fall from more than 3 meters height

- road traffic accident with
- a change in speed of delta $>30 \mathrm{~km} / \mathrm{h}$

- collision involving a pedestrian or two-wheeler

- death of a driver or passenger

- ejection of a driver or passenger

After initial management and evaluation according to the ATLS algorithm, 534 (75.8\%) received a whole-body CT according to our dedicated multiple trauma protocol. The indication for a CT scan is given by the trauma leader according to the primary clinical assessment and the trauma impact. Additionally, the indication for a whole-body CT was verified by a radiologist, since no specific protocol in terms of indications for a wholebody CT exists. The whole-body CT examination protocol starts with a head scan. After injection of intravenous contrast media it is completed by scans of the neck, thorax, abdomen and pelvis. A 64-row multidetector CT was used in all patients (Brilliance, Philips Medical Systems, Cleveland, USA). The CT scans were analysed by a trauma surgeon and a radiologist in the acute setting for injuries. Thereafter the radiologists analysed the $\mathrm{CT}$ scans in detail and wrote the report. The definitive reports were reviewed by a trauma-surgeon for Incidental Findings (IF). Correspondent to relevance IFs were subdivided into the level of concern for serious pathology they generated in the mind of the physician reading the report. Because of the expected wide variety of potential findings, three deliberately general categories were chosen according to their clinical relevance. Category 1: IF with high medical relevance with mandatory further diagnostic work-up and potential intervention prior to or shortly after hospital discharge. Examples for this category are lesions highly suspicious of malignant disease, non-trauma-associated aortic aneurysms with a diameter more than five centimeters, high-grade stenosis of major arterial vessels (>80\%), pneumonia, etc. Category 2: IF with intermediate or low medical relevance. Additional diagnostics are strongly recommended, but can be done after discharge in an out-patient setting. Examples are most likely benign lesions, aortic aneurysms with a diameter less than five centimeters. Category 3: IF without clinical relevance. Follow-up examinations or interventions are not necessary as for benign cysts of the kidney or liver. Sinusitis, mucus retention cysts, degenerative disease of the joints or vertebral column, age-related cerebral atrophy and hernias (except incarcerating hernias) were excluded.

Patients grouped into Category 1 had highly relevant findings and were considered clinically significant with acute or subacute life threatening situations. For these patients additional follow-up research was performed including Injury Severity Score (ISS), body region of 
incidentaloma and age related comorbidities. Additionally, CT reports were compared to discharge reports to verify that these findings were addressed correctly. To evaluate follow-up of the findings, hospital charts and computerized records were then reviewed to confirm that the findings were new, and to see if any follow-up investigations (histological analyses, laboratory testing, reimaging, or procedures) were subsequently performed.

Statistical analysis: Data are presented as mean values with Standard Deviation (SD \pm ) in parentheses. A Student-t or Mann-Whitney statistical test was used to compare continuous normally or not normally distributed variables, while a Chi-Square or Fisher' Exact test was used for categorical variables. Statistical analysis was performed with SPSS 20 (SPSS, Chicago, USA).

\section{Results}

534 patients were admitted over the study period and received whole body polytrauma CT as part of our trauma assessment. The mean age of the patients was 48 years (SD +/- 19.9 years, range 16-92). Gender distribution showed $377(71 \%)$ male and 157 (29\%) female patients (Table 1). 231 (43.3\%) patients were identified as having an IF. $155(67.1 \%)$ of these patients were male, 76 (32.9\%) female. A breakdown of patient acquisition is shown in Figure 1, with 231 eligible patients included in the study population. Overall, $36(6.7 \%)$ patients had incidental findings with a high clinical relevance, 48 (9.0\%) with a moderate or minor clinical relevance and 147 (27.5\%) with no clinical relevance. Interestingly all 16 patients with an ISS $\leq 10$ (3.0\%) demonstrated Category 1 incidental findings (Table 2). IFs were located in the head in $30(11.2 \%)$ patients, $16(6.1 \%)$ in the neck, 54 (20.7\%) in the thorax, $129(49.4 \%)$ in the abdomen and $32(12.3 \%)$ in the pelvis area. In 37 cases $(6.9 \%)$ more than one IF in a patient could be located in other body regions so there was a total of 497 IFs. The age distribution of patients with IFs of all categories is shown in Figure 2. The distribution of all incidental findings with respect to the three age groups, younger than 40 years, 40-60 years and older than 60 years was $10.7 \%, 14.4 \%$ and $18.2 \%$ (Figure 3 ). Referring only to IFs with moderate or high relevance a more pronounced increase was observed with age $(2.6 \%, 6.6 \%, 8.8 \%, \mathrm{p}>0.05)$. When

Table 1 Characteristics of study population

\begin{tabular}{|c|c|c|c|}
\hline \multicolumn{2}{|c|}{ Characteristics of study population: } & \multirow[t]{2}{*}{534} & \\
\hline Males & $70,6 \%(377)$ & & \\
\hline \multirow[t]{2}{*}{ Age (years) } & $48 \pm 19,78$ & (Range 16-92) & \\
\hline & $<40(n=199)$ & $40-60(n=170)$ & $>60(n=165)$ \\
\hline no IF & $142(26,6 \%)$ & $93(17,4 \%)$ & $68(12,7 \%)$ \\
\hline with IF & $57(10,7 \%)$ & $77(14,4 \%)$ & $97(18,2 \%)$ \\
\hline
\end{tabular}

Incidental findings (IF) depending on age groups. comparing patients younger than 40 years with those aged 40 or older, the difference becomes even more striking with a probability of moderately to highly relevant IFs being more than three times higher in the latter group. Absolute numbers of moderately and highly relevant IFs according to age decades can be obtained from Figure 4. For 138 (26\%) of the 534 patients receiving whole-body CT an ISS less than 16 was calculated and consequently regarded as overtriaged. Nevertheless, $12 \%$ of these showed highly relevant medical Ifs.

A detailed review of the charts of patients with Category 1 incidental findings revealed consistently poor rate of documentation of both the incidental findings and the management or referral for these lesions (Table 3). Only 19 (52.8\%) of 36 applicable Category 1 findings were mentioned in the discharge summaries. In only $10(27.8 \%)$ cases additional diagnostics, interventions or referral to a correspondent medical specialist was documented. Documentation was missing or inadequate in $47.2 \%$ of all Category 1 discharge summaries.

\section{Discussion}

Consistent with our data incidental findings in whole body CT scans for severely injured patients are very prevalent with up to $50 \%[16,17]$. We detected incidental findings in $43.3 \%$ of 534 scans with $9.0 \%$ and $6.7 \%$ classified as moderately and highly relevant in terms of clinical importance, respectively. The majority of Category 1 findings were discovered predominantly on CT scans of the abdomen (49.4\%) and the chest (20.7\%). Similar results were obtained by others analysing single CT scans of the chest or other body regions [10,11] Analogous to our results Hofstetter et al. [17] also found 6.6\% incidental findings of clinically high importance in their series of 304 multiple injured patients receiving whole-body $\mathrm{CT}$ with a consistent protocol. Incidental findings of moderate importance were described in $22.7 \%$, which is more than twice as high compared to our study cohort. This can be explained by different definitions of findings with moderate importance. Degenerative skeletal disease represented the largest portion of findings with moderate importance in the study of Hofstetter et al. [17]. Contrary, we excluded degenerative skeletal disease in our study population for two reasons. First, these diseases are very frequent, especially if mild forms are included. This potentially leads to an inappropriate positive interpretation of results. Second, degenerative disease usually has no clinical near- or midterm relevance. If at all, these diseases should be regarded as less important in our view. With a similar definition of Category 2 findings or findings of moderate clinical importance our results compare well to those of Paluska et al. [16]. On the other hand their rate of findings with high relevance was lower by approximately half with $3.1 \%$. Since they didn't use a consistent whole- 


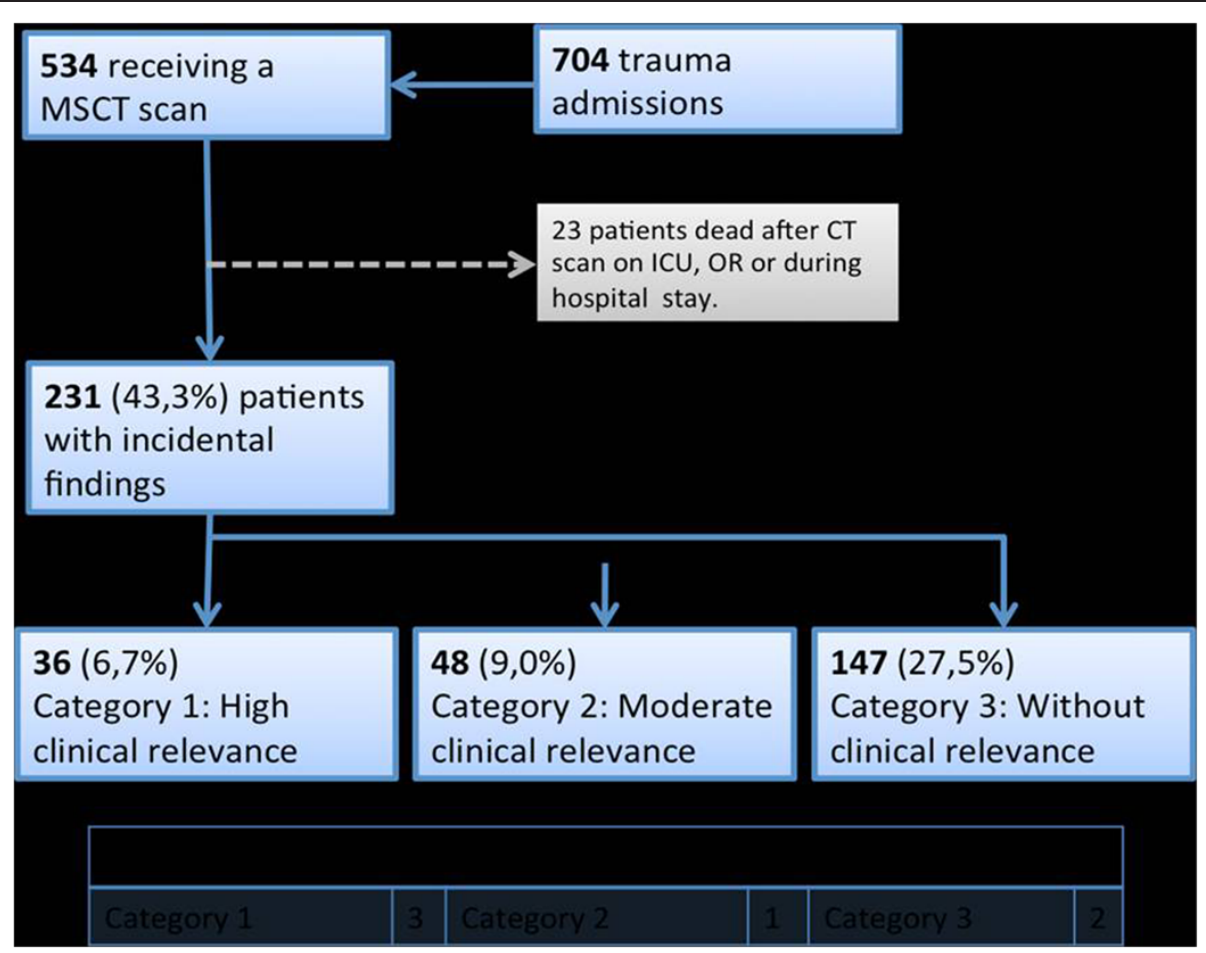

Figure 1 Flowchart demonstrating selection of study population.

Table 2 Patients with category I incidental findings and ISS $\leq 10$

\begin{tabular}{|c|c|c|c|c|c|c|}
\hline No. & Age & Gender & ISS & Reported finding & Trauma? & FU? \\
\hline 106 & 57 & $\sigma^{2}$ & 6 & Duodenal ulcer & GSI & Yes \\
\hline 238 & 46 & q & 1 & Leriche-Syndrome, adrenal adenomas & MVA & No \\
\hline 258 & 83 & q & 1 & Suspected malignant lesion in the liver and lung & Fall $<3 \mathrm{~m}$ & Yes \\
\hline 384 & 57 & $\sigma^{\top}$ & 1 & Suspected malignant lesion in the lung, Enchondroma of the Humerus & Fall $<3 \mathrm{~m}$ & No \\
\hline 391 & 71 & q & 4 & Suspected malignant lesion in the kidney & Fall $<3 \mathrm{~m}$ & Yes \\
\hline 420 & 67 & q & 8 & Suspected malignant lesion in the kidney & MVA & No \\
\hline 452 & 21 & q & 10 & Hepatomegaly with suspected malignoma & Fall $>3 \mathrm{~m}$ & No \\
\hline 464 & 38 & $\sigma^{\pi}$ & 8 & Liquefying necrotizing pneumonia & Fall $>3 \mathrm{~m}$ & Yes \\
\hline 468 & 54 & $\hat{\sigma}$ & 6 & Suspected pleural malignacy & Fall $<3 \mathrm{~m}$ & No \\
\hline 469 & 24 & q & 1 & Suspected malignant lesion in the liver, ureterolithiasis & Acerbity accident & Yes \\
\hline 474 & 69 & o & 1 & Renal cell cancer & Fall $<3 \mathrm{~m}$ & Yes \\
\hline 492 & 47 & $\hat{o}$ & 1 & Suspected lung cancer & MVA & No \\
\hline 523 & 49 & q & 4 & Suspected liver cell cancer & MVA & No \\
\hline 527 & 54 & q & 8 & Metastatic ovarian cancer & MVA & No \\
\hline 695 & 69 & q & 9 & Renal cell cancer & Fall $<3 \mathrm{~m}$ & No \\
\hline 696 & 69 & q & 4 & Suspicious complex uterus cyst, complex renal cyst, abdominal wall hernia & Fall $<3 \mathrm{~m}$ & No \\
\hline
\end{tabular}

Patients with minor or moderate Injury Severity Score (ISS $\leq 10)$ and non-trauma associated incidental findings of high relevance (category I). MVA: Motor Vehicle Accident, GSI: Gun Shot Injury, FU: Follow-Up.

$($ Q $)=$ female, $\left(\sigma^{\top}\right)=$ male. 


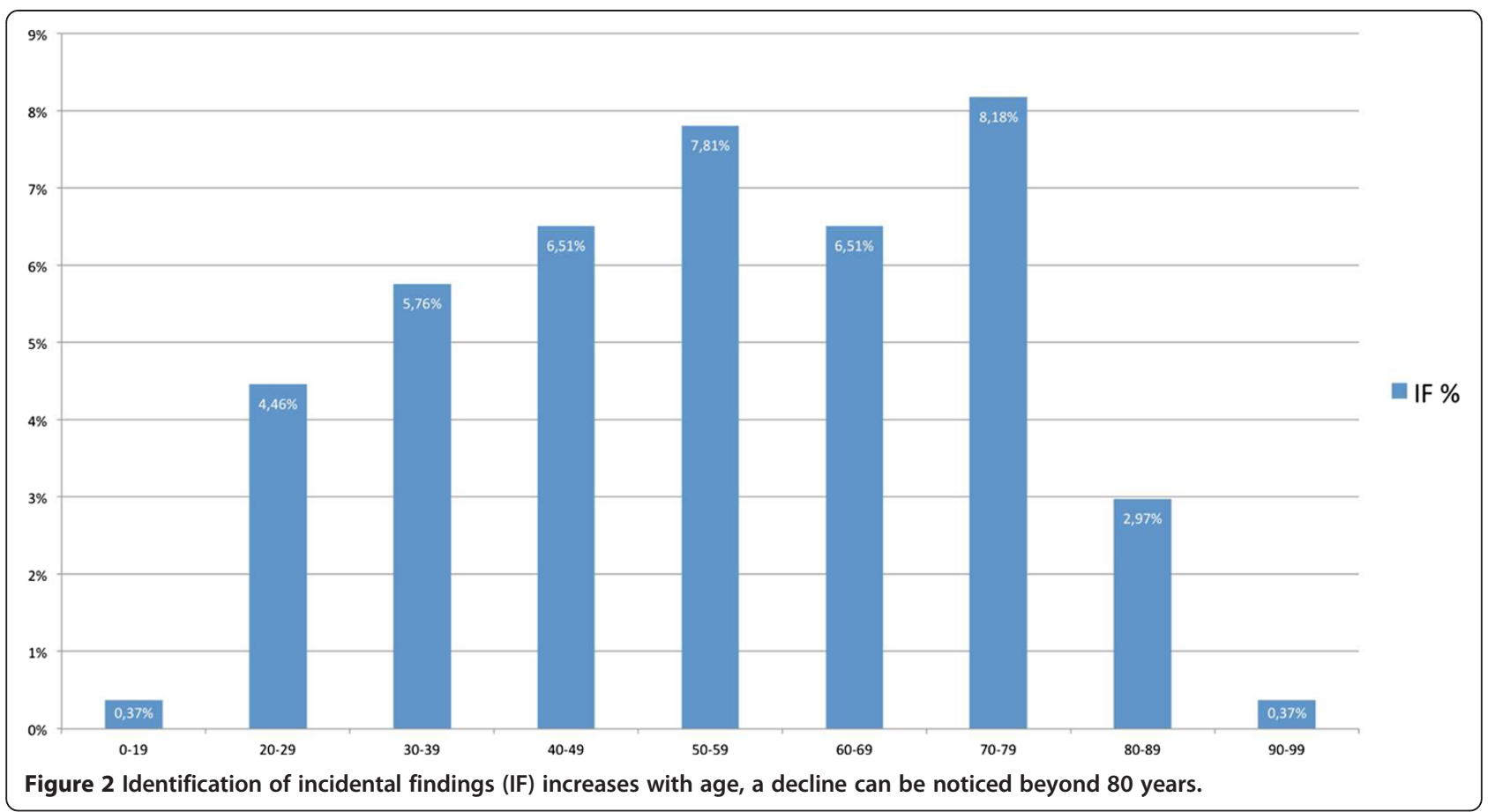

body CT protocol, but applied region specific spiral CTScans to areas of suspected injuries, many incidental findings might have been missed.

We found clinically highly important IFs in $12 \%$ of overtriaged patients with comparatively minor to moderate injuries and an ISS $<16$. The American College of Surgeons Committee on Trauma estimates that an overtriage rate of $30-50 \%$ is necessary in order to carry out efficient emergency room care [18]. Kane et al. demonstrated that in order to identify severely injured patients with a sensitivity of more than $80 \%$, the rate of overtriage could not be brought below $70 \%$ [19]. Overtriage referring to wholebody CT in trauma patients is supposed to be as high as $30-66 \%[2,20,21]$. On the other hand, implementation of whole-body CT has shown to reduce mortality in trauma patients [1]. Nevertheless, use of whole-body CT in the

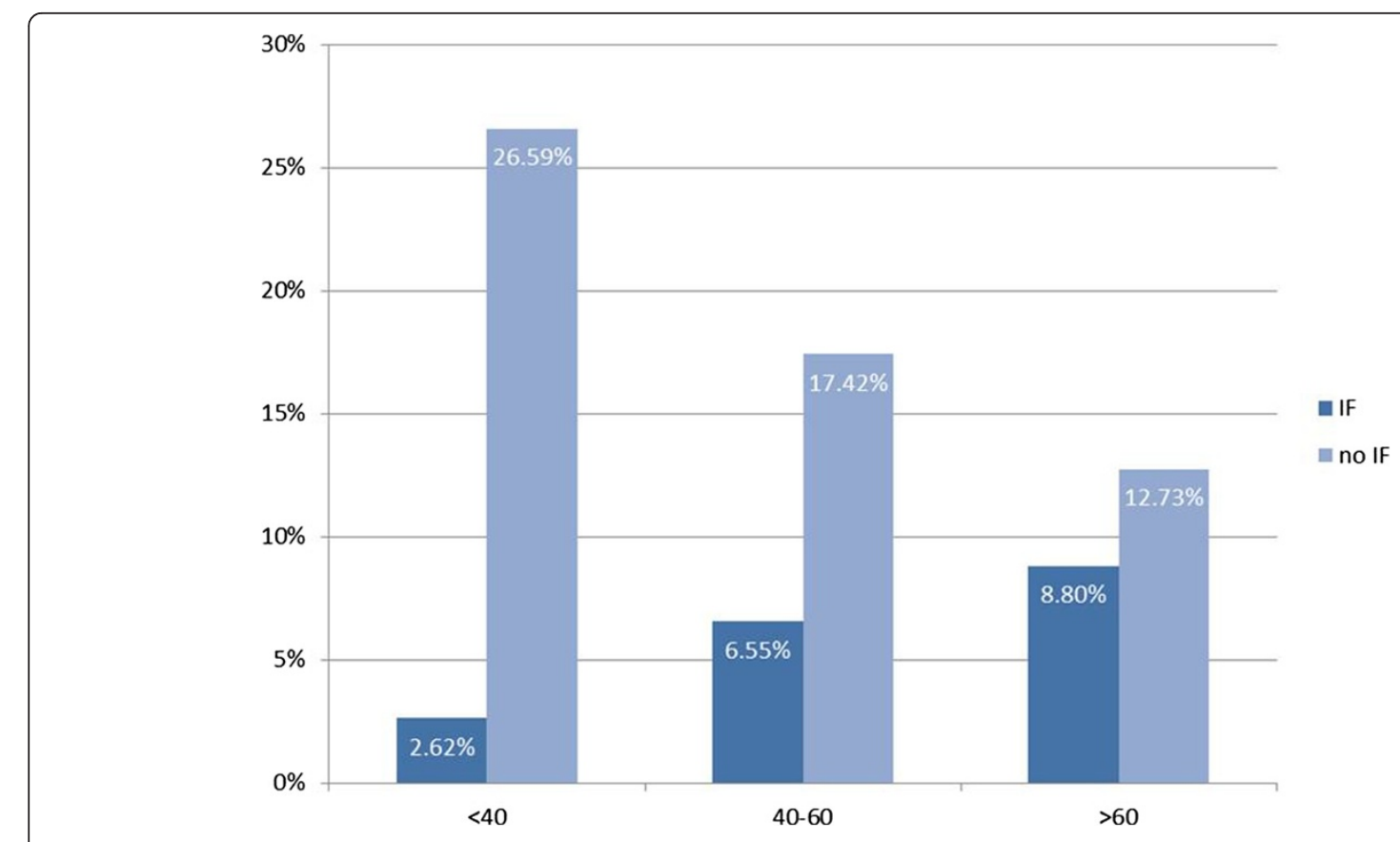

Figure 3 Incidental findings (IF) of moderate and high relevance are highest in the age group over 60 years. 


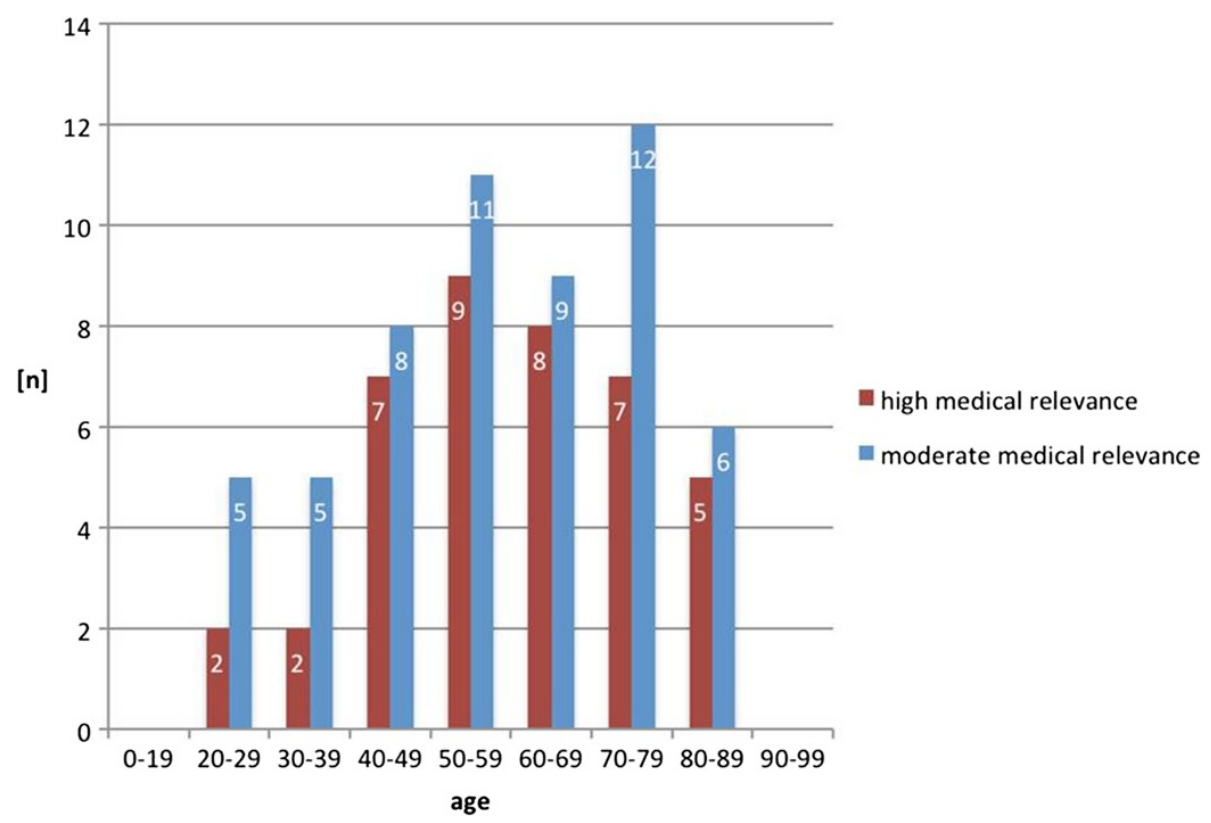

Figure 4 Incidental findings (IF) of high relevance peak in the age group 50-59 years, moderately relevant IFs show a double peak (50-59 years and 70-79 years).

assessment of trauma patients is under debate due to relatively high radiation exposure and potential indiscriminate use [22]. The radiation dose of whole-body CT is estimated to be $10-20 \mathrm{mSv}$ which results in an estimated lifetime cancer mortality for 45-year-old patient of about one in 1250 or $0.08 \%$ [13]. In our view, the overall risk of radiation exposure is outweighed by the chance of identifying possibly life threatening non-trauma associated clinical evidence in $12 \%$ of overtriaged trauma patients. Nevertheless, the trauma leader should be urged to verify the indication for whole-body CT properly, especially in patients younger than 40 years. On the other hand our study implies that whole-body CT might be used more liberally in elderly trauma patients. $40 \%$ of IFs with a moderate to severe clinical impact were found in the age group beyond 60 years in which life time cancer risks due to radiation presumably is less eminent [13]. Besides, age and comorbid conditions are independent risk factor for mortality in elderly trauma patients [23]. Additionally, minor trauma increasingly results in severe injuries among the older population [24]. Furthermore, morbidity due to complications as well as mortality is more than twice those seen in younger adult trauma patients $[24,25]$ underpinning a more liberal use of whole-body CT in the elderly.

The second key finding of this study was a poor workup of IFs with high clinical relevance. $47.2 \%$ of these findings were not documented in the discharge reports. It might be hypothesized that a growing number of elderly

Table 3 Patients with verification of incidental findings

\begin{tabular}{|c|c|c|c|c|c|c|c|}
\hline Pat. & Age & TAD & ISS & IF & $\mathrm{DL}$ & FU & Verification \\
\hline 99 & 80 & $\begin{array}{l}\text { Pertrochanteric femoral fracture, } \\
\text { floating shoulder, lung contusion }\end{array}$ & $>16$ & Suspected Bladder carcinoma & Yes & Yes & $\begin{array}{l}\text { Muscle invasive bladder } \\
\text { carcinoma, at least pT2a, G2 }\end{array}$ \\
\hline 325 & 71 & $\begin{array}{l}\text { Cervical spine fracture C6 } \\
\text { with complete paraplegia }\end{array}$ & $>16$ & Suspected Renal cell carcinoma & No & No & $\begin{array}{l}\text { Nephrectomy 09/2012: T1A } \\
\text { pNx M0 L0 V0 PNO R0 G2; }\end{array}$ \\
\hline 391 & 71 & Abdominal bleeding & 4 & $\begin{array}{l}2 \text { suspicious lesions on upper } \\
\text { kidney pole }\end{array}$ & Yes & Yes & $\begin{array}{l}\text { Nephrectomy 07/2012: pT3a(2), pNX, } \\
\text { M0, L0, V0, Pn0, UICC-Stad. III, R0 }\end{array}$ \\
\hline 392 & 34 & $\begin{array}{l}\mathrm{TB} \text {, Lumbar spine fracture } L 1 \text {, open lower leg } \\
\text { fracture, calcaneal fracture, distal radial fracture }\end{array}$ & $>16$ & $\begin{array}{l}\text { Aortic aneurysm }>5 \mathrm{~cm} \\
\text { in diameter }\end{array}$ & Yes & Yes & Stent implantation, Class. Stanford B \\
\hline 464 & 38 & Transverse process fracture of $L 1, T B \mid$ & 8 & $\begin{array}{l}\text { Suspected melted pneumonia, } \\
\text { suspicious shading of the lung }\end{array}$ & Yes & Yes & COPD with acute exacerbation \\
\hline 505 & 47 & Acetabular fracture & $>16$ & $\begin{array}{l}\text { Suspected exsudative } \\
\text { tuberculosis }\end{array}$ & Yes & Yes & $\begin{array}{l}\text { Lung TBC, pathologic changes of lung } \\
\text { tissue into squamous cell metaplasia }\end{array}$ \\
\hline
\end{tabular}

Patients with intraoperatively or histopathologically verified diagnosis of the suspected lesion in the CT-scan. TAD: Trauma associated diagnosis, ISS: Injury severity Score, IF: Incidental finding, DL: mentioned in Discharge letter, TBI: Traumatic brain injury, FU: Follow-up. 
trauma patients [9] with a high rate of concomitant comorbidities [26] increases the probability of additional unknown serious diseases as demonstrated by the high rate of major IFs in elderly patients in our study. Consequently, a potential delay in appropriate care and subsequent increased morbidity or even mortality might arise from not adequately addressed and documented IFs. Apart from ethical and socioeconomic issues, medicolegal implications must be considered in this context. Recent studies provide a potential organized approach or management pathway that can help address these difficult incidental radiographic issues [27]. The Incidental Finding Coordinator (IFC) documented incidental findings daily from trauma admission, improved notification and documentation of IFs and promoted appropriate follow-up of these patients. The implementation of an IFC resulted in more than a 2.5 -fold higher capture of incidental findings. It also resulted in an almost complete initiation of patient follow-up and hospital record documentation of incidental finding events [27].

Several limitations to this study deserve mention. It was conducted at a single level 1 trauma-center and patients may have had follow-up at another institution. Only 50\% of Category 1 findings have been verified in this study. Differences between trauma centers and detailed protocols used for diagnostic work-up and whole-body CT scans were not described. Accordingly, generalization of our results might be limited due to differences in management of trauma patients, quality of trauma care providers, and characteristics of the study sample. Regarding the diagnostic workflow, it was assumed that the radiologist reported all significant IFs. Minor findings as for degenerative skeletal diseases were not reported, subsequently our overall rate of IFs may be falsely low. Finally, no formal or verified classification of IFs is available. Therefore, a bias in determining the severity or extent of clinical impact with respect to IFs cannot be excluded.

\section{Conclusion}

Whole-body CT scans of trauma patients demonstrate a high rate of incidental findings. An incidental finding of high medical importance, potentially life-threatening, was found in approximately every 15th patient. Moreover, 9\% of these patients revealed IFs of minor to moderate medical relevance warranting further diagnostic work-up or treatment in the short- or mid-term. The diagnostic value of whole-body CT examinations are emphasized in elderly trauma patients aged 60 years or higher in which a more liberal use can be recommended in our view. Work-up and documentation of important IFs in discharge reports is poor and must be improved substantially in order to justify broader use of whole-body CT scans in elderly patients with minor or moderate trauma, as they have a higher rate of potentially life-threatening non-trauma associated new findings.

\section{Abbreviations}

CT: Computed tomography; IF: Incidental finding; ISS: Injury severity score; SD: Standard deviation; IFC: Incidental finding coordinator; TAD: Trauma associated diagnosis; TBI: Traumatic brain injury; FU: Follow-up; MVA: Motor vehicle accident; GSI: Gun shot injury.

\section{Competing interests}

The authors declare that they have no conflicting interests.

\section{Authors' contributions}

JKMF conceived the study, analyzed and interpreted the data and drafted the manuscript. OÖ acquired data of the CT and discharge protocols and helped drafting the manuscript. CJ was involved in drafting the manuscript and revising it critically for important intellectual content and gave final approval of the version to be published. All authors read and approved the final manuscript. The study was conceived without funding.

Received: 30 June 2014 Accepted: 19 August 2014

Published: 31 August 2014

\section{References}

1. Huber-Wagner S, Lefering R, Qvick ML, Körner MM, Kay MV, Pfeifer KJ, Reiser M, Mutschler W, Kanz KG, Working Group on Polytrauma of the German Trauma Society: Effect of whole-body CT during trauma resuscitation on survival: a retrospective, multicentre study. Lancet 2009, 373:1455-1461.

2. Wurmb TE, Quaisser C, Balling H, Kredel M, Muellenbach R, Kenn W, Roewer $\mathrm{N}$, Brederlau J: Whole-body multislice computed tomography (MSCT) improves trauma care in patients requiring surgery after multiple trauma. Emerg Med J 2011, 28:300-304.

3. Stengel D, Frank M, Matthes G, Schmucker U, Seifert J, Mutze S, Wich M, Hanson B, Giannoudis PV, Ekkernkamp A: Primary pan-computed tomography for blunt multiple trauma: can the whole be better than its parts? Injury 2009, 40(Suppl 4):36-46.

4. Rieger M, Sparr H, Esterhammer R, Fink C, Bale R, Czermak B, Jaschke W: Modern CT diagnosis of acute thoracic and abdominal trauma. Radiologe 2002, 42:556-563.

5. Kalra MK, Rizzo SMR, Novelline RA: Technologic innovations in computer tomography dose reduction: implications in emergency settings. Emerg Radiol 2005, 11:127-128.

6. Philipp MO, Kubin K, Hörmann M, Metz VM: Radiological emergency room management with emphasis on multidetector-row CT. Eur J Radiol 2003, 48:2-4.

7. Latifi A, Torkzad O, Labruto F, Ullberg U, Torkzad MR: The accuracy of focused abdominal CT in patients presenting to the emergency department. Emerg Radiol 2009, 16:209-215.

8. Nguyen D, Platon A, Shanmuganathan K, Mirvis SE, Becker CD, Poletti P-A: Evaluation of a single-pass continuous whole-body 16-MDCT protocol for patients with polytrauma. AJR Am J Roentgenol 2009, 192:3-10.

9. Broder J, Warshauer DM: Increasing utilization of computed tomography in the adult emergency department, 2000-2005. Emerg Radiol 2006, 13:25-30.

10. Richman PB, Courtney DM, Friese J, Matthews J, Field A, Petri R, Kline JA: Prevalence and significance of nonthromboembolic findings on chest computed tomography angiography performed to rule out pulmonary embolism: a multicenter study of 1,025 emergency department patients. Acad Emerg Med 2004, 11:642-647.

11. Messersmith WA, Brown DF, Barry MJ: The prevalence and implications of incidental findings on ED abdominal CT scans. Am J Emerg Med 2001, 19:479-481.

12. Shrimpton $P C$, Wall BF: CT-an increasingly important slice of the medical exposure of patients. Br J Radiol 1993, 66:1067-1068.

13. Brenner DJ, Elliston CD: Estimated radiation risks potentially associated with full-body CT screening. Radiology 2004, 232:735-738.

14. Salim A, Sangthong B, Martin M, Brown C, Plurad D, Demetriades D: Whole body imaging in blunt multisystem trauma patients without obvious signs of injury: results of a prospective study. Arch Surg 2006, 141:468-473. discussion 473-5.

15. Snyder GE: Whole-body imaging in blunt multisystem trauma patients who were never examined. Ann Emerg Med 2008, 52:101-103. 
16. Paluska TR, Sise MJ, Sack DI, Sise CB, Egan MC, Biondi M: Incidental CT findings in trauma patients: incidence and implications for care of the injured. J Trauma 2007, 62:157-161.

17. Hoffstetter P, Herold T, Daneschnejad M, Zorger N, Jung EM, Feuerbach S, Schreyer AG: Non-trauma-associated additional findings in whole-body CT examinations in patients with multiple trauma. Rofo 2008, 180:120-126.

18. American College of Surgeons Committee on Trauma: Resources for the Optimal Care of the Injured Patient. Chicago: American College of Surgeons; 1998.

19. Kane G, Wheeler NC, Cook S, Englehardt R, Pavey B, Green K, Clark ON, Cassou J: Impact of the Los Angeles County Trauma System on the survival of seriously injured patients. J Trauma 1992, 32:576-583.

20. Wurmb TE, Frühwald P, Hopfner W, Keil T, Kredel M, Brederlau J, Roewer N, Kuhnigk H: Whole-body multisclice computed tomography as the first line diagnostic tool in patients with multiple injuries: the focus on time. J Trauma 2009, 66:658-665.

21. Linsenmaier U, Kanz KG, Rieger J, Rock C, Pfeifer K, Reiser M: Structured diagnostic imaging in patients with multiple trauma. Radiologe 2002, 42:533-540

22. Weninger P, Mauritz W, Fridrich P, Spitaler R, Figl M, Kern B, Hertz H: Emergency room management of patients with blunt major trauma: evaluation of the multislice computed tomography protocol exemplified by an urban trauma center. J Trauma 2007, 62:584-591.

23. Woischneck D, Peters B, Kapapa T, Skalei M, Firsching R: Comparison of younger and elder patients after multiple trauma. Versicherungsmedizin 2013, 65:4-8.

24. Giannoudis PV, Harwood PJ, Court-Brown C, Pape HC: Severe and multiple trauma in older patients; incidence and mortality. Injury 2009, 40:362-367.

25. Schiller WR, Knox R, Chleborad W: A five-year experience with severe injuries in elderly patients. Accid Anal Prev 1995, 27:167-174.

26. Wutzler S, Maegele M, Marzi I, Spanholtz T: Association of preexisting medical conditions with in-hospital mortality in multiple-trauma patients. J Am Coll Surg 2009, 209:75-81.

27. Sperry JL, Massaro MS, Collage RD, Nicholas DH, Forsythe RM, Watson GA, Marshall GT, Alarcon LH, Billiar TR, Peitzman AB: Incidental radiographic findings after injury: Dedicated attention results in improved capture, documentation, and management. Surgery 2010, 148:618-624.

Cite this article as: Fakler et al:: Retrospective analysis of incidental non-trauma associated findings in severely injured patients identified by whole-body spiral CT scans. Patient Safety in Surgery 2014 8:36.

\section{Submit your next manuscript to BioMed Central and take full advantage of:}

- Convenient online submission

- Thorough peer review

- No space constraints or color figure charges

- Immediate publication on acceptance

- Inclusion in PubMed, CAS, Scopus and Google Scholar

- Research which is freely available for redistribution 